\title{
Interpréter les comédies de Térence : Aelius Donat et l'actio
}

Sabine Chaouche

\section{(2) OpenEdition}

1 Journals

Édition électronique

URL : http://journals.openedition.org/rhetorique/560

DOI : $10.4000 /$ rhetorique.560

ISSN : 2270-6909

Éditeur

UGA Éditions/Université Grenoble Alpes

Édition imprimée

ISBN : 978-2-37747-029-7

Référence électronique

Sabine Chaouche, «Interpréter les comédies de Térence : Aelius Donat et l'actio », Exercices de

rhétorique [En ligne], 10 | 2017, mis en ligne le 26 décembre 2017, consulté le 21 décembre 2020. URL :

http://journals.openedition.org/rhetorique/560 ; DOI : https://doi.org/10.4000/rhetorique.560

Ce document a été généré automatiquement le 21 décembre 2020.

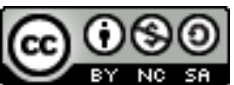

Les contenus de la revue Exercices de rhétorique sont mis à disposition selon les termes de la Licence Creative Commons Attribution - Pas d'Utilisation Commerciale - Partage dans les Mêmes Conditions 4.0 International. 


\title{
Interpréter les comédies de Térence : Aelius Donat et l'actio
}

\author{
Sabine Chaouche
}

Dans un article consacré à Eugraphius, Sarah Laborie souligne :

Le commentaire d'Eugraphius est très largement consacré à l'inuentio, et reprend amplement les préceptes des artes rhetoricae des écoles de rhétorique antiques ou tardo-antiques, tandis que celui de ses collègues [Aelius Donat, Servius, et Tiberius Donat] est beaucoup plus tourné vers l'elocutio, l'étude des tropes et des figures rhétoriques, bref, vers un savoir typique des grammatici ${ }^{1}$.

Se penchant plus spécifiquement sur le travail d'Aelius Donat ${ }^{2}$, elle affirme: «Le commentaire grammatical que pratique couramment un Aelius Donat le conduit à s'attacher à la lettre du texte: lorsqu'il explicite une métaphore ou qu'il décrit un archaïsme, le commentateur cherche avant tout à faire comprendre le sens des mots qu'emploie Térence ${ }^{3} »$. Donat, à la différence d'Eugraphius, a privilégié une étude philologique et didactique des textes de Térence, s'attachant plus particulièrement au fonctionnement de la langue latine et au style. Le grammairien, dont les commentaires sont "fragmenté[s] et lemmatisés ${ }^{4}$ », ainsi qu' une succession de scholies souvent juxtaposées sans principe organisateur ${ }^{5}$ ", semble donc ignorer délibérément l'action dramatique en elle-même, autrement dit l'inventio, de même que la représentation du texte, c'est-à-dire l'actio. Or, doit-on penser que les scholies de Donat omettent réellement toute référence à la dramaturgie ou à la scène dans la mesure où le commentaire relève d'une démarche rhétorique? Si Bruno Bureau et Christian Nicolas ont montré que l'argumentation de Donat abordait la fiction vraisemblable au théâtre symbolisée par les intrigues de la comédie, à travers notamment le motif de la parole efficace ${ }^{6}$, peu a été dit sur la manière dont Donat analyse l'œuvre de Térence et donne une interprétation de la manière dont les pièces devraient être représentées ${ }^{7}$. En ce sens, Chrysanthi Demetrious a récemment montré que les commentaires présentaient un caractère multidimensionnel étant destinés à un large public ${ }^{8}$. Ils offrent, de fait, une vue d'ensemble sur l'œuvre de Térence, «allant des techniques de rhétorique à l'analyse de l'action comique " ". À l'aune de ce constat, il convient d'examiner en quoi les notes sur l'éloquence du corps témoignent, chez Donat, d'une forme de conception 
embryonnaire de mise en scène. Les scholies, parce qu'elles relèvent à la fois du champ de la rhétorique et du jeu de l'acteur, posent la question de la nature et de la fonction des informations données par le grammairien. S'agit-il de simples commentaires, ou au contraire, d'indications scéniques, et donc de sortes de didascalies établies à partir du texte? Quels sont dès lors leurs liens avec les règles de l'actio établies par Quintilien et doit-on considérer que Donat applique à la lettre ces principes ou au contraire qu'il donne sa propre interprétation des comédies de Térence, prenant appui sur des témoignages anciens ou contemporains relatifs au théâtre à Rome ? La lecture critique des pièces permet d'expliciter le contenu du texte tout en le liant à sa mise en scène comique. Si les commentaires montrent l'omniprésence de principes rhétoriques destinés à guider l'acteur, à travers les explications données sur l'actio, qu'elle soit considérée comme défectueuse ou non dans l'art oratoire, ils révèlent également que l'auteur va au-delà du texte, en proposant sa propre conception de la scène, laquelle va de pair avec ce que l'on pourrait qualifier de jeu réaliste de l'acteur.

\section{Des indications intra-textuelles sans commentaires?}

D'après C. Demetriou, «le commentateur semble s'adresser à ses lecteurs de façon instructive, en expliquant comment une réplique ou un mot spécifique doit être dit ${ }^{10}$ ». Cette remarque souligne le fait que les comédies de Térence sont explicitées par Donat, sans doute parce qu'il s'adresse en premier chef à des étudiants. Ces derniers s'exercent de façon pratique en cultivant l'art de la disputatio ou plus probablement en interprétant les dialogues de Térence ${ }^{11}$. Il n'est pas sûr pour autant que les comédies de celui-ci furent systématiquement représentées du vivant de Donat, quand bien même il serait encore, avec Plaute, considéré comme un auteur majeur au $\mathrm{IV}^{\mathrm{e}}$ siècle $^{12}$. Art théâtral et rhétorique sont depuis longtemps intimement liés et les échanges entre les deux disciplines, fréquents. Quintilien souhaite ainsi dans son Institution oratoire qu'un acteur professionnel vienne enseigner l'art oratoire dans les écoles ${ }^{13}$ et que certains passages de pièces de théâtre soient sélectionnés s'ils sont semblables au discours de l'orateur ${ }^{14}$.

Cependant on peut d'emblée souligner que de nombreux gestes décrits ou suggérés par Donat appartiennent au bas comique et à des types ${ }^{15}$ dont le caractère est grossier, ainsi de l'esclave ou du parasite, personnages incontournables de la comédie latine, et de la courtisane présente dans L'Hécyre. Leur comportement traduit l'excès, pourtant fermement rejeté dans l'art oratoire préférant la médiocrité, c'est-à-dire l'équilibre. Basore relève par exemple des gestes ou expressions qui ne sont pas forcément liés à l'art oratoire mais plutôt au jeu théâtral, c'est-à-dire à la mimique, comme le " geste de sortie [gestus exeuntis vel abituri] ${ }^{16}$ ", le "geste qui donne à voir ce que l'on pense [gestus cogitantis] ${ }^{17}$ ", le " geste de menace [gestus comminantis] ${ }^{18}$ ", de même que la présence de gesticulation et de mouvements violents reflétant de fortes émotions ${ }^{19}$, ou de gestes vus comme déplacés dans l'art oratoire comme tousser ou secouer la tête, et même jubiler d'excitation ${ }^{20}$. Les grimaces sont elles aussi abordées. Certaines émotions très marquées sur le visage des personnages contrastent avec les préceptes de l'actio, l'orateur devant garder son calme et maîtriser son ethos.

Dès lors, on peut s'interroger sur la relation établie entre les constituants du texte théâtral, notamment les répliques comiques pourtant a priori peu conformes à la 
gravitas et à l'auctoritas requises chez un orateur ${ }^{21}$, et leur actualisation par Donat, autrement dit sur les indications intra-textuelles à partir desquelles se fondent les analyses du grammairien qui, en choisissant Térence, semble indiquer que certaines scènes sont adaptées à l'enseignement de l'actio (notamment du point de vue de la récitation ou déclamation), ou tout du moins que l'étude de la scène comique a toute sa place dans la formation de l'orateur.

Donat s'attache à cinq pièces de Térence, les notes relatives à L'Heautontimoroumenos ayant été perdues. Les comédies de Térence comportent elles-mêmes assez peu d'indications internes. Les didascalies sont quant à elles absentes. Térence semble ainsi suivre les préceptes d'Aristote sur le texte dramatique, les informations concernant la mise en scène et le jeu de l'acteur devant apparaître au cœur du texte. Si l'on prend le cas de L'Andrienne, on retrouve ainsi dans l'écriture même des procédés visant à souligner l'actio. Certains sont évidents, assurant la liaison entre les scènes (« Fort à propos voici Dave, dont les conseils font mon appui ${ }^{22}$ ", II, 1). Marquant les entrées et sorties des personnages, ils seront systématiquement exploités au XVII ${ }^{\mathrm{e}}$ siècle. Les comédies ne sont pas exemptes de références aux réactions des personnages ou à leur propre langage corporel. Ainsi de cette indication scénique : «Ah! ne t'emporte pas $\operatorname{tant}^{23} »(\mathrm{~V}, 2)$, ou de cette autre doublée d'une description de l'état du personnage : « Mais que vois-je ? Pamphile tout hors de lui ${ }^{24}$ » $(\mathrm{I}, 4)$ ou de cette réplique de Mysis en aparté lorsqu'elle observe Pamphile et met l'accent sur l'agitation du jeune homme, de même que sur l'effet que celle-ci provoque en elle : «Je tremble de voir sur quoi ce "je ne sais" va déboucher ${ }^{25}$ » $(\mathrm{I}, 5)$. Les répliques sont parfois d'ailleurs redondantes et surchargent le texte comme en témoigne cet extrait de la scène 3 de l'acte $\mathrm{V}$ : «Charinus. - Qu'est-ce que cette joie ? Pamphile. - C'est Dave que je vois. Personne qui ne convienne mieux ; car je sais qu'il est le seul à pouvoir si bien jouir de ma joie ${ }^{26}$. " Ces indications apparaissent superflues dans la mesure où le spectateur est à même de réaliser, à travers l'actio sur scène, ce que ressentent les personnages. Ces éléments du texte représentent en soi des indications scéniques qui sont elles-mêmes par nature des commentaires puisqu'elles visent essentiellement à décrire la passion ou l'éloquence du corps. Enfin, certains mouvements ou au contraire l'absence de mouvements sont précisés. À la fin de la comédie, Pamphile demande à Dave de partir : «Hé ! Pourquoi ne bouges-tu pas? Qu'attends-tu?». Sur quoi répond l'esclave: "J'y vais. Pour qu'on n'attende pas qu'ils sortent et reviennent ici. C'est à l'intérieur que se feront les accords et les arrangements, s'il en reste à faire ${ }^{27}$. " D'une part, Dave est invité à sortir de scène et d'autre part, ce dernier indique aux spectateurs que la pièce est terminée et qu'il ne faut donc plus s'attendre à voir qui que ce soit sur scène. En outre, l'usage de l'adjectif démonstratif est aussi révélateur, comme par exemple lorsqu'il suggère un geste de la main ("C'est dans cette rue, m'a-t-on dit, qu'habitait Chrysis ${ }^{28}$ ", IV, 5). Finalement, il existe également un certain nombre de gestes, de mouvements ou d'émotions décrits par les personnages, notamment dans les récits («Elle met la main de Glycère dans la mienne, et la mort aussitôt la prend ${ }^{29}$ ", I, 5 ou «Aussitôt je cours en toute hâte à la place pour te porter cette nouvelle. Comme je ne te trouve pas, je monte sur un endroit élevé, je regarde autour de moi : personne ${ }^{30}$ ", II, 2), ainsi que des remarques relatives à l'ethos ( Quand tu vois sa figure il n'a pas l'air d'un homme de rien. Il a une gravité austère sur le visage, et dans ses paroles de la bonne foi ${ }^{31}$ ", V, 2) et même la comédie jouée par un personnage à un autre ("Comme si tu étais surpris, retourne-toi vers lui ${ }^{32}$ ", II, 5). 
Toutes ces indications intra-textuelles qui mériteraient une étude plus poussée, sans doute évidentes pour le lecteur, ne sont nullement commentées par Donat alors qu'elles pourraient donner matière à discussion, notamment sur le fonctionnement du dialogue et l'utilisation de la langue pour mettre en valeur implicitement l'actio. Or les commentaires de Donat portent précisément sur d'autres aspects du texte qu'il est nécessaire de prendre en compte en ce qu'ils servent de didascalies - et non pas seulement de simples commentaires. Comme le remarque Marika Thomadaki dès l'introduction de son article sur les mises en scène de Térence, «Les auteurs anciens nous ont légué leurs textes démunis d'indications aux lecteurs et aux metteurs en scène » mais Donat a su trouver « une foule de signes ${ }^{33}$ » au cœur du texte.

\section{Les commentaires sur l'actio: scholies ou didascalies?}

Si l'on reprend le cas de L'Andrienne à titre comparatif, on note par exemple l'explicitation de gestes qui ne sont pas directement mentionnés dans le texte et qui peuvent être propres au type (" Dave se glisse sur la scène, en faisant de nombreuses mimiques à l'intention des spectateurs ${ }^{34}$ », I, 2), à la situation sans qu'il soit forcément aisé de comprendre la signification même du geste choisi ( «Il faut prononcer cela de manière démonstrative, en se touchant l'oreille par exemple $\left.{ }^{35} »\right)$. Donat indique de manière récurrente les mimiques ${ }^{36}$. Ainsi certains mots sont "éclairés " comme le souligne Donat lui-même, par le jeu de l'acteur ou ne sont destinés qu'au jeu de l'acteur : « Ici c'est la gestuelle sur scène qui met le mieux en valeur la réplique, de fait ces mots sont écrits plus pour des spectateurs que pour des lecteurs " affirme le grammairien ${ }^{37}$, suggérant, de fait, que certaines parties du texte sont plus 'dramatiques' car destinées aux jeux de scène ou jeux de physionomie ${ }^{38}$ - et donc a fortiori ne relevant pas de l'actio oratoire -, que d'autres, plus 'rhétoriques' - et donc plus adaptées aux disputes organisées dans les écoles.

Les remarques sur la prononciation sont quant à elles focalisées sur l'emphase devant accompagner certains mots ou groupes de mots: "il dit erus et pater sur un ton grandiloquent ${ }^{39} »$. Les commentaires sur la diction rejoignent majoritairement les règles de l'actio, cette partie de la rhétorique comportant de nombreuses règles vocales destinées à ornementer et mettre en valeur le discours, quelles que soient les émotions exprimées. L'analyse des passions et la manière de les dire sur scène relèvent bien ainsi de cette "mécanique » de la voix qui a un impact fort sur l'auditoire lorsqu'elle est savamment maîtrisée $e^{40}$, et qui sera, plus tard, si souvent commentée par les rhétoriciens au cours de la première modernité. Elle se compose de l'accent oratoire, de l'accent des passions et de la prononciation des mots ou lettres (pronuntiatio). Donat fait donc une analyse lorsqu'il établit la manière de prononcer une réplique et lorsqu'il se réfère à la situation dramatique afin de déterminer la tonalité des paroles devant être dites. Ainsi par exemple dans L'Andrienne: "VT MEMOR ESSES SVI sur un ton agressif, comme si elle parlait à un homme sans mémoire : c'est pour cela que Pamphile est si bouleversé par ces mots ${ }^{41}$ ", ou "MEA GLYCERIVM INQVIT QVID AGIS mea (ma chère), comme dirait un amoureux, Glycerium, comme dirait un familier, quid agis comme dirait un homme profondément troublé, cur te is perditum comme dirait un homme qui console ${ }^{42}$ ». L'utilisation de «comme si » ou du conditionnel ${ }^{43}$ montre qu'il s'agit bien ici d'un travail d'interprétation et d'explicitation des répliques. Toutefois, Donat dépasse le 
simple cadre de l'analyse rhétorique. En effet, s'il détermine le sens du texte et les divers segments à prononcer avec insistance ou non, ou rapidement ou non ${ }^{44}$, voire de façon articulée ou non ${ }^{45}$, il souligne également les silences ou respirations nécessaires dans le discours ${ }^{46}$, se comportant ainsi comme un véritable directeur de scène, voire un acteur. Il peut lier geste et prononciation, expliquant à la fois ce que présuppose la construction même du texte (autrement dit l'organisation des répliques) et ce qu'elle implique en termes d'actio, ainsi de ces deux scholies sur la répétition de mots qui montrent à la fois le raisonnement de Donat et la conclusion à laquelle il parvient en matière de jeu scénique :

1 HEM QVID PAMPHILI apparet mulierem uestri et Pamphili lentius pronuntiasse quam Dauus uellet, et ideo hunc repetisse nomen nostri ?, Pamphili ? 2 HEM QVID duas res operatur Dauus simul : et ut ignarissimum se ostendat et ut Pamphilus nominetur. et argute repetit nomen Pamphili quasi per indignationem, ut soceri auribus adulescentis nomen inculcet; metuit enim, ne non audiat uelut senex.

1 HEM QVID PAMPHILI il est clair que la femme a prononcé les termes uestri et Pamphili à voix plus basse que Dave ne l'aurait voulu, et que par conséquent il reprend le nom nostri ?, Pamphili ?. 2 HEM QVID Dave travaille à deux choses en même temps : il agit de manière à se montrer totalement ignorant et de manière à ce que Pamphile soit nommé. Et astucieusement il répète le nom de Pamphile comme sous le coup de l'indignation, pour faire pénétrer le nom du jeune homme dans les oreilles du beaupère ; il a eu peur en effet qu'il n'entende pas vu que c'est un vieillard ${ }^{47}$.

Étonnamment, Donat ne met pas en valeur les indications intra-textuelles insérées dans les comédies, mais au contraire ajoute des didascalies qui révèlent certains jeux de scène et qui semblent traduire une volonté de transmettre des traditions théâtrales, le plus souvent des jeux de scène impliquant mouvements et grimaces. Ainsi, de même qu'il était important d'aborder la nature des scholies et ce à quoi elles se réfèrent, il semble désormais nécessaire de voir en quoi Donat ouvre la voie à une interprétation du texte.

\section{Visualiser la scène : vers une actio figurative ?}

Dans son ouvrage sur les commentaires de Donat publié en 1908, J. W. Basore montre de façon exhaustive que de nombreux gestes sont tirés de l'art oratoire parce qu'ils relèvent de la convention ${ }^{48}$ (notamment les défauts de l'orateur), et que, de fait, scène théâtrale et art oratoire ont des points de convergence. Il établit la liste de tous les mouvements et gestes rencontrés dans les pièces de Térence ${ }^{49}$, notamment ceux de la tête, des mains et des doigts ${ }^{50}$. En outre, les passions et émotions, centrales dans l'art oratoire, sont relevées et divisées en deux catégories ${ }^{51}$ : d'une part les expressions faciales, extrêmement nombreuses, qui laissent à penser que l'acteur joue sans masque $^{52}$ (elles concernent les sourcils ${ }^{53}$, les lèvres ${ }^{54}$ et les yeux ${ }^{55}$, parties du visage détaillées par Quintilien dans son Institution oratoire lorsqu'il souligne l'importance pour l'orateur d'avoir un visage mobile capable de manifester les passions de l'âme ${ }^{56}$ ); d'autre part, la démonstration des émotions ${ }^{57}$ comme le rire $^{58}$, la joie ${ }^{59}$, le mécontentement ${ }^{60}$, les sanglots ${ }^{61}$ ou la manière dont le visage peut changer au gré des réactions ${ }^{62}$. De nombreux gestes et expressions commentés par Donat donnent ainsi des informations sur la mise en scène, autrement dit servent à illustrer visuellement le texte comme par exemple dans L'Andrienne, lorsque Pamphile et Simon montrent le sac d'argent que tient Chrémès ${ }^{63}$. Cette visualisation est encore à l'œuvre dans Les Adelphes lorsque Donat enjoint le lecteur de se représenter « les gestes du locuteur à partir de 
ses paroles ${ }^{64}$ ». Donat tente donc de faire du lecteur un spectateur, recréant la mise en scène mais faisant également appel à l'imagination et au bon sens du lecteur afin qu'il voit, en lui-même, le jeu de l'acteur.

Toutes ces expressions enrichissent les scholies et permettent de faire émerger une forme d'actio figurative, reflétant les réactions et les sentiments des personnages. Donat décrit les expressions faciales, mais se veut le plus souvent prescriptif ${ }^{65}$, donnant sa propre vision de la manière dont un personnage agit ou réagit sur scène. Peu de commentaires restent en effet ouverts ${ }^{66}$. Il impose ainsi une manière de jouer que l'on pourrait qualifier de péremptoire, comme s'il avait lui-même assisté aux représentations ou devenait lui-même directeur de scène.

Selon J.W. Basore, «bien que Donat n'ait pas nécessairement vu les comédies représentées sur scène, il n'est pas impossible qu'il ait pu vouloir les annoter en incluant des indications scéniques en fonction de ses propres idées, soit pour la lecture à haute voix ou sans volonté réelle de les mettre en pratique ${ }^{67} »$. Pourtant il apparaît que certaines remarques dépassent le simple cadre de l'analyse de texte, témoignant d'une connaissance technique de la scène ou tout du moins d'une volonté réelle de faire comprendre au lecteur la mise en scène. On relève ainsi dans L'Andrienne de nombreuses notes sur le fonctionnement même de la scène et l'occupation de ses différents espaces ${ }^{68}$, les coulisses et l'avant-scène ( on va désormais en coulisse, parce que par la suite plus rien ne se passe à l'avant-scène $\left.{ }^{69} »\right)$, les placements (« Il faut noter que les jeunes filles de naissance libre ne prennent jamais la parole sur le devant de la scène dans une comédie palliata ${ }^{70} »$, « il se tourne désormais vers le public, avec lequel il a commencé sa tirade $\left.{ }^{71} »\right)$, la relation entre l'organisation de l'espace et les mouvements des personnages ( il nous faut en revanche garder en tête que Térence fait mention dans le début du voisinage des deux maisons pour nous faire comprendre qu'un personnage peut entrer et sortir d'un mouvement très discret $\left.{ }^{72} »\right)$, les entrées et les sorties des personnages qui délimitent les actes ( $«$ ces mots sont ceux de quelqu'un qui revient du marché ${ }^{73}$ ", " Dave entre en scène en exultant et en annonçant qu'il n'y a plus aucun danger ${ }^{74}$ ", «ici Criton entre en scène $\left.{ }^{75} »\right)$. Cette démarche permet de visualiser mentalement la scène sans avoir recours nécessairement à l'illustration ${ }^{76}$ et fait des commentaires une forme embryonnaire de manuscrit de souffleur ${ }^{77}$.

L'hypothèse avancée par Basore est que Donat se serait servi de manuscrits ayant appartenu à des troupes et rendus accessibles par des savants latins dont les travaux portaient sur les arts du spectacle à Rome, ou de copies d'acteurs, et aurait sélectionné les indications scéniques les plus pertinentes ${ }^{78}$. Il ajoute, en outre, que Donat montre un intérêt manifeste pour l'art de la représentation, ce dont témoigne effectivement le $D e$ Fabula d'Évanthius consacré à la dramaturgie mais également au costume ${ }^{79}$ et à l'histoire des spectacles. Donat estime qu'une des qualités de Térence est d'avoir su mettre en scène des personnages figuratifs et vraisemblables ${ }^{80}$. L'idée de mimèsis semble dès lors centrale, d'où la référence directe à Cicéron définissant le genre comique comme étant « une image du réel » (« La comédie est, selon Cicéron, une imitation de la vie, un miroir de l'usage, une image du réel $\left.{ }^{81} »\right)$. Les commentaires visent précisément à mettre en valeur ce réalisme tant du point de vue des gestes que des paroles prononcées par l'acteur ${ }^{82}$, dans la mesure où ils constituent l'essence même de la représentation théâtrale comique : «La comédie, du fait qu'elle est un poème composé sous couleur d'imiter la vie et de décalquer les mœurs, consiste en gestes et en paroles ${ }^{83}$ ». Ce souci de vraisemblance explique dans doute pourquoi Donat prend le soin de 
relever certaines traditions théâtrales qui ne paraissent pas ou plus réalistes comme par exemple le bonnet d'Ulysse porté par les acteurs ou le diadème d'Achille et Néoptolème ${ }^{84}$.

En définitive, les commentaires de Donat ne font pas forcément référence à des passages comparables au discours de l'orateur. Très souvent, le grammairien interprète la dramaturgie et la situation scénique, allant au-delà de son rôle de commentateur. Les éléments scéniques qu'il relève reflètent sa volonté d'assurer une meilleure compréhension de l'intrigue en fonction des personnages mis en scène, notamment leur caractère ou type, et de leurs interactions, autrement dit de la situation dramatique. Ils sont donc englobés dans l'examen dramaturgique des pièces et en ce sens, deux niveaux de lecture semblent être possibles: l'un qui tire du texte des références implicites à l'actio qu'il s'agit de formuler pour les rendre explicites ; l'autre qui appréhende la représentation théâtrale dans sa globalité à travers la mise en scène. Donat fait ainsi tout autant figure de rhétoricien que de "directeur de scène ». Cette double fonction se révèle dès lors intéressante dans la mesure où l'art dramatique sera soumis, au cours de la première modernité, à l'influence de l'art oratoire. On peut gager que certains acteurs ayant suivi des cours de rhétorique comme Molière ou Corneille par exemple, pouvaient eux aussi penser de façon plus ou moins technique la mise en scène de leurs propres pièces, à la manière de Donat, ou que cette formation rhétorique, notamment l'initiation à l'analyse du texte dramatique, se révélait utile pour diriger des acteurs ou se développer en tant qu'acteur ${ }^{85}$, comme il apparaît par exemple pour Henri Louis Lekain dont les manuscrits témoignent de ce besoin d'interpréter le texte pour mieux le comprendre et ainsi construire son personnage de la façon la plus aboutie.

\section{NOTES}

1. S. Laborie, «Le commentaire d'Eugraphius aux comédies de Térence », Études littéraires, vol. 43, $\mathrm{n}^{\circ} 2,2012$, p. 31 .

2. Nous ne nous engageons pas ici dans un débat sur les différents états du texte et les manuscrits de Donat, sur les variantes et sur la précision ou non des scholies. D'après Michael Reeve, reconstituer la version première des commentaires s'avère impossible (M. D. Reeve, "Aelius Donatus ", dans Texts and Transmission: A Survey of the Latin Classics, éd. L. D. Reynolds, Oxford, Clarendon Press, 1983, p.153-156). Enfin, nous ne discuterons pas non plus la paternité des commentaires qui nous sont parvenus puisqu'ils sont une compilation des scholies provenant de différentes versions. Sur l'histoire des commentaires, voir $\mathrm{Ch}$. H. Beeson, « The Text Tradition of Donatus's Commentary on Terence ", Classical Philology, 17, n 4, oct. 1922, p. 283-305, J. N. Grant, Studies in the Textual Tradition of Terence, Toronto, University Press, 1986 et J. A. Barsby, « Donatus on Terence: The Eunuchus Commentary » dans Dramatische Wäldchen: Festschrift für Eckard Lefèvre zum 65. Geburtstag, éd. E. Stärk and G. Vogt-Spira, Hildesheim, Olms, 2000, p. 491-513.

3. Laborie, op. cit., p. 31.

4. Ibid., p. 32. 
5. Ibid., p. 37 : «Le commentaire d'Aelius Donat, de fait, ne prétend pas au titre d'œuvre, mais représente un corpus mouvant, enrichi à plusieurs occasions, au fil des différents manuscrits, de corrections et de marginalia ».

6. B. Bureau et $\mathrm{C}$. Nicolas, "Argumentum et fiction dramatique dans le commentaire de Donat à Térence", dans Théorie et pratique de la fiction à l'époque impériale, éd. C. Bréchet, A. Videau, R. Webb, Paris, Éditions Picard, «Textes, images et monuments de l'Antiquité au Haut Moyen Âge ", 2013, p. 99-122.

7. Voir M. Thomadaki, «La mise en scène du théâtre de Térence dans le commentaire de Donat ", Dioniso, 59, 1989, p. 365-372.

8. C. Demetriou, «Aelius Donatus and His Commentary on Terence's Comedies », dans The Oxford Handbook of Greek and Roman Comedy, éd. M. Fontaine et A. C. Scafuro, Oxford, University Press, 2014, p. 782-800. Le présent article doit beaucoup aux travaux de C. Demetriou qui est également l'auteure d'une thèse sur le sujet: Donatus's Commentary on Terence: A study of the Scholia on Performance, Université de Leeds, 2011.

9. Demetriou, «Aelius Donatus and His Commentary... », op. cit., p. 784 (« ranging from techniques of rhetoric to analyses of comic action ", nous traduisons, ici et ensuite).

10. Ibid., p. 784 ("The commentator often seems to be addressing readers in an instructive way, explaining how a line or specific word should be delivered »).

11. Aelius Donat fut professeur et maître de Saint Jérôme. Son Ars Donati Grammatici Urbis Romae fut publié à l'intention d'élèves, et se divisait en deux parties (cours élémentaire et cours avancé). Selon Louis Holtz, Donat serait resté célèbre parce qu'il aurait été un bon pédagogue. Voir L. Holtz, Donat et la tradition de l'enseignement grammatical. Étude sur l'Ars Donati et sa diffusion (IVe-IX siècle) et édition critique, Paris, CNRS, 1981, p. 90-93.

12. J. W. Basore, The Scholia on Hypokrisis in the Commentary of Donatus, Baltimore, J. H. Furst, 1908, p. 2.

13. Quintilien, Institutio Oratoria (Inst. Orat.), I, 11, 4 (texte établi et traduit par Jean Cousin, Paris, Les Belles Lettres, 1979).

14. Quintilien, Inst. Orat., I, 11,12-13.

15. On retrouve des références directes à ces types bien spécifiques dans : And., 184, 4 (« quid hic vult correspond à l'éthos de l'esclave et au jeu du bouffon; en effet, c'est ainsi que se parlent à eux-mêmes, avec un jeu de physionomie de circonstance, les esclaves de comédie appelés par leur maître »); Eun., 274, 3 ( "Vro hominem il se parle à lui-même en prenant le visage et la mimique typiques du parasite. »). - Les références aux pièces de Térence et aux commentaires de Donat sont tirées du site internet Hyperdonat: B. Bureau, C. Nicolas, M. Ingarao, Aelii Donati in Hecyram Terenti commentum, Hyperdonatus - Editiones collectae antiquorum commentorum electronicae cum translatione, commentariis et adnotationibus criticis, 2012, http://hyperdonat.tge-adonis.fr/. Abréviations: L'Andrienne (And.), L'Eunuque (Eun.), Les Adelphes (Ad.), L'Hécyre (Héc.), Le Phormion (Phorm.).

16. J. W. Basore, op. cit., p. 7. Par exemple : Eun., 549, 1 (« NVM QVIS HIC EST NEMO EST in hac scaena uerba gestum uultumque indicant exeuntis, cui obuia persona obicitur, sub cuius occasione spectatoribus gesta narrabuntur "; "NVM QVIS HIC EST NEMO EST dans cette scène, les mots indiquent l'attitude et l'expression de celui qui sort qui tombe nez à nez avec l'autre personnage ; c'est à cette occasion que ses actions seront racontées aux spectateurs. »).

17. Par exemple : Ad., 265, 4-5 (désappointement : « et simul gestum considera loquentis ex uerbis. [...] mire hoc uerbo apparet in uultu lenonis et spem mortuam et restinctum gaudium "; "Et en même temps, représentez-vous les gestes du locuteur à partir de ses paroles [...] de façon remarquable, avec ce mot apparaît sur le visage du proxénète la fin de ses espérances et la disparition de sa gaieté »). Voir également Ad., 536, 1 (moquerie : «MEAS TVAS et meas et tuas cum gestu pronuntiare debemus, ut intellegatur irrisio "; " nous devons prononcer et meas et tuas avec un geste, pour faire comprendre la moquerie ».) ; And., 415 (épier : « in gestu est, nam est figura corporis obseruantis, quid 
agatur »; « c'est dans la gestuelle, de fait, il faut représenter un personnage en train d'observer ce qui va se passer »).

18. Par exemple: And.,753, 2 (menace: «CAVE: comminatus est et uultu, ut suspenderet comminationem; deinde infert gestum »; «il a menacé aussi avec son visage, pour suspendre la menace ; ensuite il ajoute le geste »).

19. Par exemple Phorm., 350, 5-6 (exaspération menant à une injonction : « annuit, ut taceat ; Et significat silentium »; « il fait un signe pour qu'il se taise ; et l'expression signifie silence. ») ; Eun., 771, 1 (colère: " hic rursus inepti uanitas militis demonstratur ad amicam tamquam ad hostilem exercitum pergentis irritato animo, concito cursu, undanti chlamyde, trepidi et quatientis caput. "; « dans cette scène, on montre de nouveau la vanité du stupide soldat qui court vers sa maîtresse comme vers une armée ennemie, l'esprit bourru, au pas de course, la chlamyde ondoyante, tout frémissant et secouant la tête. »); And., 183, 1 (" hoc et gestu et uultu seruili et cum agitatione capitis dixit »; « en disant cela, il fait les mimiques et les grimaces de l'esclave, il secoue la tête »).

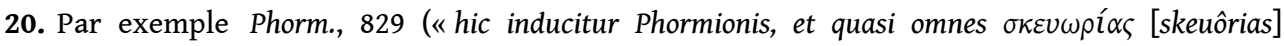
prouenisse laetatur »; "la vantardise de Phormion est ici représentée, et il en va comme s'il se

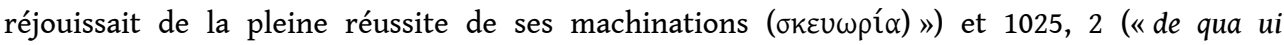
contradictionis exsultat parasitus tamquam confecto mortuoque aduersario suo "; « la violence de cette réplique remplit le parasite de joie, comme si son adversaire était achevé et mort »). Également dans Ad., 265, 3 («oportet autem men quaerit cum quadam gesticulatione et subsaltatione pronuntiari sperantis lenonis ad hoc se quaeri, ut accipiat »; «Il faut alors prononcer men quaerit avec les gestes et les sursauts de celui qui espère qu'on le cherche pour lui donner quelque chose »).

21. Notons que Quintilien évoque le cas de la comédie, utile selon lui pour l'instruction de jeunes garçons en ce qu'elle montre les diverses émotions agitant l'âme humaine ainsi que différents caractères (Inst. Orat., I, 8, 7: «Comoediae, quae plurimum conferre ad eloquentiam potest, cum per omnes et personas et adfectus eat, quem usum in pueris putem, paulo post suo loco dicam; nam cum mores in tuto fuerint, inter praecipua legenda erit. " "Quant à la comédie, qui peut apporter beaucoup à l'éloquence, puisqu'elle traite de toutes sortes de caractères et de sentiments, je vais dire bientôt, à la place qui convient, l'usage qu'on peut en faire, selon moi, pour l'instruction des enfants ; car, dès qu'il n'y aura plus à craindre pour leur moralité, elle devra figurer au nombre de leurs lectures principales. »

22. «Dauum optume uideo, cuius consilio fretus sum » (335).

23. « ah ne saeui tanto opere» (868).

24. « sed quidnam Pamphilum exanimatum uideo?» (234).

25. « timeo 'incertum' hoc quorsum accidat » (264).

26. «Ch. - quid illud gaudi est? P. - Dauum uideo; nemo est quem mallem omnium; nam hunc scio mea solide solum gauisurum gaudia » (963-964).

27. «P.- em quid stas, quid cessas ? D. - eo. ne exspectet dum exeant huc: intus despondebitur; intus transigetur si quid est quod restat » (979-981).

28. « in hac habitasse platea dictum est Chrysidem » (796).

29. " hanc mihi manum dat; mors continuo ipsam occupat " (297).

30. " continuo ad te properans percurro ad forum ut dicam haec tibi / ubi te non inuenio ibi escendo in quemdam excelsum locum, / circumspicio : nusquam » (355-357).

31. «cum faciem uideas, uidetur esse quantiuis pretii ; tristis seueritas inest in uoltu atque in uerbis fides " (856-857)

32. « quasi de improuiso respice ad eum » (417).

33. Thomadaki, op. cit., respectivement p. 365 et 366.

34. " hic locus est, in quo Dauus insinuatur spectatoribus multa gesturus» (And. 175).

35. "demonstratiue ueluti aurem suam tangens" (And. 186). Ce signe est, d'après Basore, destiné à raviver la mémoire. C'est un geste commun dans l'Antiquité lorsque l'on veut qu'une personne témoigne lors d'un procès. 
36. And., 226, 232.

37. " hic gestu scaenico melius commendatur, nam haec magis spectatoribus quam lectori scripta sunt " (And. 310).

38. Par exemple And., 348, 350, 363, 415, 722.

39. « erum et patrem cum ingenti pronuntiatione dicit » (And., 867). Voir également: And., 102 (« PLACVIT DESPONDI Cum pronontiatione placuit »; «PLACVIT DESPONDI dans la prononciation, insistez sur placuit »), 360 (« Et de improuiso et nuptiae pronuntiandum »; « il faut souligner par la prononciation de improviso et nuptiae.»), 625 («Et totum in pronuntiatione est : hoc enim genus interrogationis uim negandi exprimit "; "Et tout est dans la prononciation car ce genre d'interrogation exprime la valeur négative »), 719 («cum admiratione pronuntia quem et cum exclamatione quadam»; "prononcez quem avec admiration et avec une forme d'exclamation»), 810 (« Me cum emphasi »; » Me est dit avec emphase »).

40. Voir Aristote, Politique, livre VIII, 7 (sur les effets du chant) et le Pseudo-Aristote, Problèmes, $\mathrm{n}^{\circ} 19$ (sur les sons), ainsi que Quintilien, Inst. Orat., XI, 3.

41. And., 281, 5: «VT MEMOR ESSES SVI inuidiose quasi oblito: adeo commouetur hoc dicto Pamphilus ».

42. And., 134: « MEA GLYCERIVM INQVIT QVID AGIS mea quasi amator, hoc glycerium quasi familiaris dixit, quid agis quasi perturbatus, cur te is perditum quasi consolatus ".

43. Voir également: And., 469, 1 (« ADEON EST DEMENS EX PEREGRINA Ě $\lambda \lambda \varepsilon \imath \psi \imath \varsigma$ per $\dot{\alpha} \pi O \sigma \iota \omega ́ \pi \eta\rceil \imath$ [elleipsis per aposiôpèsin] apta cogitanti. reliquum autem sic pronuntiat quasi reperto consilio "; " ADEON EST DEMENS

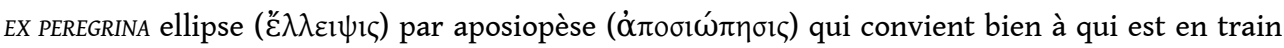
de réfléchir. Le reste, en revanche, il le prononce comme s'il avait trouvé une idée ») ou 709, 3 (« $a b$ eo hoc quasi ab iocante pronuntiandum est, nam sic ille impudens respondet "; " Il doit prononcer cela comme s'il plaisantait ; de fait, c'est sur ce ton que l'autre répond impudens ») et Eun., 451 (« AT MIHI ISTVC NON IN MENTEM VENERAT Sic pronuntiandum est, quasi militi monstri simile uideatur sapiens dictum alii prius uenisse in mentem quam sibi »; « AT MIHI ISTVC NON IN MENTEM VENERAT il faut prononcer comme si c'était pour le soldat l'équivalent d'un prodige que de voir une parole sage venir à l'esprit d'un autre avant lui »).

44. Par exemple : And., 920 («SI MIHI PERGIT QVAE VOLT DICERE grauiter et mature »; «SI MIHI PERGIT QVAE VOLT DICERE sur un ton grave et dans un tempo rapide ») et 922, (" IAM SCIRI POTEST addidit et temporis celeritatem et magna maturitate dixit »; «IAM SCIRI POTEST il ajoute une grande promptitude et le dit avec un tempo rapide $»)$.

45. Eun., 282, 2 (« QVIA ISTAM DVCIS hoc quasi ad Gnathonem, sed lente ac sub lingua murmurat "; " QVIA ISTAM DVCIS ces mots sont adressés pour ainsi dire à Gnathon, mais c'est un murmure circonspect et presque inarticulé »).

46. And., 958, 1 ( NVNC SIC ESSE HOC VERVM LVBET sic delicate pronuntiandum est et separatim distinguendum »; « NVNC SIC ESSE HOC VERVM LVBET il faut prononcer sic de façon délicate et le détacher avec une pause » et 938, 1 («Et $\dot{\alpha} \sigma v v \delta \dot{\varepsilon} \tau \omega \varsigma$ [asundetôs] distinguendum est spe gaudio, ut separatim inferatur mirando tanto hoc tam repentino bono "; "Et il faut une ponctuation forte avec asyndète

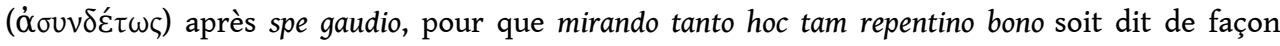
détachée du reste »).

47. And., 765, 1 et 2 .

48. J. W. Basore, op. cit., p. 10.

49. Voir Quintilien, Inst. Orat., XI, 379 : «Vitium in superciliis si aut inmota sunt omnino aut nimium mobilia aut inaequalitate, ut modo de persona comica dixeram, dissident aut contra id quod dicimus finguntur: ira enim contractis, tristitia deductis, hilaritas remissis ostenditur. Adnuendi quoque et renuendi ratione demittuntur aut adleuantur.» («Les défauts dans les sourcils, c'est l'immobilité totale ou l'excès de mobilité ou leur irrégularité discordante, comme sur le masque de comédie dont je viens de parler, ou le contraste de leur expression avec les paroles qui sont dites : en effet, 
froncés, ils expriment la colère, abaissés la tristesse, détendus la joie. De même, pour dire oui ou non, on les baisse ou on les hausse. »).

50. Quintilien (Inst. Orat., XI, 3, 71) distingue les gestes de l'orateur de ceux des histrions : « Significat uero plurimis modis. Nam praeter adnuendi, renuendi, confirmandique motus sunt et uerecundiae et dubitationis et admirationis et indignationis noti et communes omnibus. Solo tamen eo facere gestum scaenici quoque doctores uitiosum putauerunt. Etiam frequens eius nutus non caret uitio: adeo iactare id et comas excutientem rotare fanaticum est. " ("La tête a d'ailleurs de multiples modes d'expression. Car, outre les mouvements d'acquiescement, de refus et de confirmation, il y en a aussi pour la honte, et le doute, et l'étonnement, et l'indignation, qui sont connus et communs à tous les hommes. Cependant, borner le geste à la tête a été regardé comme un défaut même par les maîtres d'art dramatique. Faire de fréquents signes de tête est aussi un défaut; à plus forte raison, l'agiter souvent, et la tourner en secouant la chevelure est d'un fou furieux. »).

51. J. W. Basore, op. cit., p. 43-56. Basore suggère que le mot "vultu » est employé de manière récurrente par Donat, quand bien même il resterait général.

52. Des débats se sont tenus sur le sujet : certains critiques ont estimé que Donat se fondait sur des traditions propres à la scène térentienne; d'autres ont contesté cette approche. Pour une mise au point sur la question, voir C. Demetriou, « Aelius Donatus and His Commentary... », op. cit. et G. S. Aldrete, Gestures and Acclamations in Ancient Rome, Baltimore, Johns Hopkins University Press, 1999.

53. Par exemple : Phorm., 184, 4 (« ERVS ADEST uultu tristi ac superciliis arduis hoc dicendum est »; « ERVS ADEST il faut dire cela avec un visage sombre et en fronçant les sourcils »); Hec., 468, 1 (« OMNEM REM SCIO VT SIT GESTA Pamphilus uultuose et cum supercilii tristitia hoc dicit »; » OMNEM REM SCIO VT SIT GESTA Pamphile dit cela avec une mimique et un froncement de sourcil triste. »).

54. Par exemple: Hec., 748, 4 (« SOCRVS terribiliter quasi quae maxime sciat, quae maxime doleat. ergo et oculis et labris pronuntiandum "; "SOCRVs dit pour faire peur, comme celle qui en sait le plus et qui a le plus à en souffrir. Il faut donc dire ce mot tant avec les yeux qu'avec les lèvres ») ; Phorm., 315, 3 ( ITANE PATRIS AIS CONSPECTVM VERITVM HIC ABISSE Haec labra lingens ut ebrius et ructans utpote et satur pronuntiauit actor bonus »; " ITANE PATRIS AIS CONSPECTVM VERITVM HIC ABISSE En bon acteur, il a prononcé ces mots en se mouillant les lèvres comme un ivrogne et en rotant comme quelqu'un qui est complètement ivre. »).

55. Par exemple : EUn., 974 ( « SED ESTNE ILLE NOSTER PARMENO ET CERTE IPSVS et dubitauit et affirmauit postea utpote senex uisu iam languido atque oculis propter aetatem defessis »; «SED ESTNE ILLE NOSTER PARMENO ET CERTE IPSVS EST en même temps il doute puis il affirme après coup comme un vieillard dont la vue a déjà baissé et dont les yeux sont fatigués par l'âge »); Ad., 96, 2 ("NVLLVM HVIVS SIMILE hoc cum admiratione indignantis est pronuntiandum et ardentibus in Micionem oculis »; " NVLLVM HVIVS SIMILE il faut dire cela avec l'étonnement d'un homme en colère et des yeux ardents dardés contre Micion »).

56. Quintilien, Inst. Orat., XI, 3, 72 :» Dominatur autem maxime uultus. Hoc supplices, hoc minaces, hoc blandi, hoc tristes, hoc hilares, hoc erecti, hoc summissi sumus; hoc pendent homines, hunc intuentur, hic spectatur etiam antequam dicimus; hoc quosdam amamus, hoc odimus, hoc plurima intellegimus, hic est saepe pro omnibus uerbis.»; "Le rôle souverain est surtout dévolu au visage. Par son intermédiaire, nous sommes suppliants, par lui menaçants, par lui flatteurs, par lui tristes, par lui gais, par lui fiers, par lui humbles; c'est à lui qu'est suspendu l'auditoire, sur lui que se fixe l'attention, lui qu'on regarde, même avant que nous parlions, lui qu'on aime, lui qu'on hait, lui qui nous fait entendre beaucoup de choses, lui qui vaut souvent toutes les paroles ».

57. La plupart des gestes relatifs à la tête visent à ajouter du sens au discours comme par exemple consentir ou refuser. Bien entendu, secouer la tête, en revanche, reste un geste scénique.

58. Par exemple: Ad., 844, 1 (" EO PACTO PRORSVS ILLIC ALLIGABIS FILIVM haec cum riso dicit Micio »; «EO PACTO PRORSVS ILLIC ALLIGABIS FILIVM Micion dit cela en riant. ») ; Eun., 1002, 2 (« Ergo persona cachinnans 
inducitur quaerens Parmenonem aspectuque eius ob nimium risum uix loqui posse "; " Donc il met en scène un personnage qui pouffe de rire en cherchant Parménon et qui en le voyant s'étrangle de rire au point d'à peine pouvoir parler. »).

59. Eun., 1031, 1 and 2, (« POPVLARES ECQVIS ME VIVIT HODIE FORTVNATIOR hic persona Chaereae est feruens gaudio ut adulescentuli et in prosperis rebus. 2. [...] non mirum est, si insane exsultat nuptiis, qui iamdudum ob id solum, quod uitiauerat uirginem, laetabatur"; "POPVLARES ECQVIS ME VIVIT HODIE FORTVNATIOR ici le personnage de Chéréa est tout bouillant de joie, comme il convient à un petit jeune homme, qui plus est au milieu d'événements heureux. 2. [...] il n'est pas étonnant de le voir sauter de joie comme un fou à l'idée de son mariage, lui qui depuis longtemps déjà se réjouissait du seul fait qu'il avait déshonoré la jeune fille »).

60. Par exemple: And., 306, 1 (« NIHIL VOLO ALIVD NISI PHILVMENAM hoc magis gemitu amatorio quam ut responderet Byrrhiae »; " NIHIL VOLO ALIVD NISI PHILVMENAM cela est plus un gémissement d'amant qu'une réplique visant à répondre à Byrria »);Hec., 263, 4 (« LACHES ET DILIGENTIAM VESTRAM Et cum affectu quodam et gemitu dixit Laches"; « LACHES ET DILIGENTIAM VESTRAM. Et il dit Laches avec une sorte de passion et un gémissement »).

61. Par exemple : Héc., 385, 1 (« NEQVEO QVIN LACRIMEM MISER uidetur quasi destillantes fletus detergere »; « NEQVEO QVIN LACRIMEM MISER il semble presque essuyer ses pleurs qui coulent »). Voir J. W. Basore, op. cit., p. 56-61.

62. Par exemple: Eun., 304, 1 (« QVID TV ES ALACRIS alacritas est mutatio quaedam uultus gestientis in spem aliquam "; " QVID TV ES ALACRIS le mot alacritas désigne quelque changement d'expression du visage qui s'agite dans quelque espoir. »).

63. And., 950, 1 (« NEMPE SCILICET nempe et scilicet dicentes manu uel uultu dotem significant, quod mox intellegit Chremes"; "NEMPE SCILICET en disant nempe (à savoir) et scilicet (évidemment), ils désignent de la main ou du regard la dot, ce que comprend Chrémès peu après »).

64. Ad., 265, 4 : " gestum considera loquentis ex uerbis ".

65. Donat utilise abondamment la tournure prescriptive «il faut». Les exemples sont très nombreux et concernent principalement la prononciation : And., 28, 5 (« ABITE concitatius legendum est, quia respectantes properat et discernit a Sosia »; "ABITE il faut prononcer ce mot avec une certaine rapidité, car il presse les serviteurs qui regardent en arrière et les sépare de Sosie »); Ad., 149, 1 (« QVAM HIC NON AMAVIT MERETRICEM AVT CVI NON DEDIT [...] ea tamen haec sunt, ut non graui sono accusationis proferantur, sed solam reprehensionem contineant »; " QVAM HIC NON AMAVIT MERETRICEM AVT CVI NON DEDIT [...] pour autant, il ne faut pas prononcer cela avec le ton grave de l'accusateur, mais en marquant seulement du reproche »); 199, 3 (« HOMINI MISERO PLVS QVINGENTOS COLAPHOS flebiliter pronuntiandum : hoc enim exprimitur incusare eum in alieno facto fortunam suam »; « HOMINI MISERO PLVS QVINGENTOS COLAPHOS il faut le dire sur un ton pleurnichard») et 214, 4 (« ADVLESCENTI MOREM GESTVM cum emphasi adulescenti pronuntiandum »; " ADVLESCENTI MOREM GESTVM il faut prononcer adulescenti avec emphase ») etc.

66. Eun., 171, 1 (« OB HAEC FACTA ABS TE SPERNOR hic duplex pronuntiatio est : uel per interrogationem uel per inuidiosam exprobrationem »; «OB HAEC FACTA ABS TE SPERNOR ici, double prononciation possible : ou sur le mode interrogatif ou sur celui de l'exclamation indignée. »).

67. J. W. Basore, op. cit., p. 3 (« though Donat may not have witnessed the presentation of comedies upon the stage, it is not unbelievable that he may have been inclined to annotate such with stage directions according to his own ideas, either for purposes of reading aloud or with no practical intent »).

68. And., Praefatio, I, 3 : « sed illa re plerumque decipimur, quod personam, cum tacuerit, egressam falso putamus, quae nihilo minus in proscaenio tacens loquendi tempus exspectat. est igitur attente animaduertendum, ubi et quando scaena uacua sit ab omnibus personis [...]» ("Souvent nous nous trompons en pensant à tort qu'un personnage, du fait qu'il était silencieux, vient d'entrer en scène, alors qu'il attend sans parler à l'avant-scène le moment pour lui de donner une réplique. Il faut donc observer attentivement où et quand la scène se vide de tous les personnages [...]»). 
69. And., 978, 2: « iam post scaenam itur, quia ulterius in proscaenio nihil agitur ». Voir également Eun., 391, 1 (« MAGNAS VERO AGERE GRATIAS THAIS MIHI hic sermo sic prodit, ut post scaenam incohatus esse noscatur »; " MAGNAS VERO AGERE GRATIAS THAIS MIHI. Cette conversation se présente de telle sorte qu'on comprend qu'elle a débuté en coulisses »).

70. And., Praefatio, I, 10: « adnotandum sane puellarum liberalium in proscaenio nullam orationem induci in comoedia palliata $[. .]$.$» .$

71. And., Prologus, 24, 1: « iam ad auditores conuertitur, a quibus coepit».

72. And., Praefatio, III, 6: « sed tenere debemus ideo Terentium uicinitatis mentionem fecisse in principio, ut modico receptu et adesse et abesse personam intellegamus ». Cf. Eun., 232, 1 (« DI INMORTALES HOMINI HOMO QVID PRAESTAT in hac scaena non stans sed quasi ambulans persona inducitur; constitit tamen aliquantum intuens spectatores, dum secum loquitur »; "DI INMORTALES HOMINI HOMO QVID PRAESTAT dans cette scène on représente un personnage non pas statique, mais pour ainsi dire en déplacement ; toutefois il s'arrête de temps en temps et regarde les spectateurs quand il se parle à lui-même »).

73. And., 745, 1: « haec uerba sunt uenientis de foro ».

74. And., 842, 2: « exsultans egreditur Dauus nuntians prona esse omnia ad securitatem ».

75. And., 904, 1: « hic in scaenam progreditur Crito ».

76. Certains manuscrits de Térence furent en effet accompagnés d'illustrations. Voir les analyses de C. Demetriou sur le sujet, op. cit., p. 789-794.

77. Les annotations présentes dans les manuscrits de souffleur, telles qu'on les retrouve à l'époque prémoderne et moderne, sont toujours relatives aux placements, aux déplacements de l'acteur, au décor, et à la manière d'interpréter le texte. Elles concernent rarement l'aspect lexical ou stylistique d'une réplique.

78. J. W. Basore, op. cit.., p. 4-5.

79. Evanthii de Fabula (consultable en ligne: http://hyperdonat.huma-num.fr/editions/html/ DonEva.html), VI, 5 et VIII, 6-8.

80. Ibid., III, 4 .

81. Ibid., V, 1 (« comoediam esse Cicero ait imitationem uitae, speculum consuetudinis, imaginem ueritatis »). Voir dans ce même numéro, l'article de Bruno Bureau.

82. Cf. ibid., III, 8 ( " illud quoque mirabile in eo, primo quod non ita miscet quattuor personas, ut obscura sit earum distinctio, et item quod nihil ad populum facit actorem uelut extra comoediam loqui, quod uitium Plauti frequentissimum "; «il est remarquable aussi d'abord qu'il ne mélange pas quatre personnages sans qu'on puisse facilement les distinguer et aussi qu'il ne fait jamais parler au public un acteur, comme s'il sortait de la comédie, défaut très fréquent chez Plaute. »).

83. Ibid., V, 3 : «Comoedia autem, quia poema sub imitatione uitae atque morum similitudine compositum est, in gestu et pronuntiatione consistit ».

84. Ibid., VIII, 4, sur Ulysse: "Vlixen pilleatum semper inducunt, siue quod aliquando insaniam simulauit, quo tempore tectum se esse uoluit, ne agnitus cogeretur in bella prodire, seu ob singularem sapientiam, qua tectus munitusque plurimum sociis profuit; huius enim uirtus erat animi semper decipientis ingenium. nonnulli Ithacae incolas sicut Locros pilleatos fuisse commemorant "; "On met toujours en scène Ulysse avec un bonnet, soit parce qu'à un moment il a simulé la folie, et qu'alors il voulait rester couvert pour éviter d'être reconnu et envoyé à la guerre, soit à cause de son extraordinaire intelligence dont il se couvrait et se protégeait et fit beaucoup profiter ses alliés; car sa qualité résidait dans le génie de son esprit toujours prêt à tromper. Certains rappellent que les habitants d'Ithaque, comme ceux de Locres, portaient des bonnets ». Voir aussi VIII, 5 sur Achille et Neoptolème: "Achillis et Neoptolomi personae diademata habent, quamuis numquam regalia sceptra tenuerunt. cuius argumenti probatio talis inducitur, quod numquam cum reliqua Graeciae iuuentute ad gerenda cum Troianis bella sacramenta coniurationis inierunt nec umquam sub Agamemnonis imperio fuerunt.»; "Les personnages d'Achille et de Néoptolème portent des diadèmes, quoiqu'ils n'aient jamais tenu de sceptre royal. Pour justifier cet état de fait, on avance 
que, au contraire du reste de la jeunesse grecque, ils n'ont jamais prêté le serment conjoint de faire la guerre aux Troyens et n'ont jamais été sous le commandement d'Agamemnon ».

85. Sur cet aspect particulier voir S. Chaouche, La Philosophie de l'Acteur, la dialectique de l'intérieur et de l'extérieur dans les écrits sur l'art théâtral français, 1738-1801, Paris, Honoré Champion, 2007, $2^{\mathrm{e}}$ partie.

\section{AUTEUR}

\section{SABINE CHAOUCHE}

The University of Oxford \& Sunway University 